\title{
Identification of Symmetry-Protected Topological States on Noisy Quantum Computers
}

\author{
Daniel Azses $\odot,{ }^{1,2}$ Rafael Haenel@, ${ }^{3,4}$ Yehuda Naveh, ${ }^{5,}$ Robert Raussendorf $\odot,{ }^{3,4}$ \\ Eran Sela, ${ }^{6}$ and Emanuele G. Dalla Torre ${ }^{1,2}$ \\ ${ }^{1}$ Department of Physics, Bar-Ilan University, Ramat Gan 5290002, Israel \\ ${ }^{2}$ Center for Quantum Entanglement Science and Technology, Bar-Ilan University, Ramat Gan 5290002, Israel \\ ${ }^{3}$ Department of Physics and Astronomy, University of British Columbia, Vancouver, British Columbia V6T 1Z1, Canada \\ ${ }^{4}$ Stewart Blusson Quantum Matter Institute, University of British Columbia, Vancouver, British Columbia V6T 1Z4, Canada \\ ${ }^{5}$ IBM Research-Haifa, Haifa University Campus, Mount Carmel, Haifa 31905, Israel \\ ${ }^{6}$ School of Physics and Astronomy, Tel Aviv University, Tel Aviv 6997801, Israel
}

(Received 17 May 2020; accepted 14 August 2020; published 14 September 2020)

\begin{abstract}
Identifying topological properties is a major challenge because, by definition, topological states do not have a local order parameter. While a generic solution to this challenge is not available yet, a broad class of topological states, namely, symmetry-protected topological (SPT) states, can be identified by distinctive degeneracies in their entanglement spectrum. Here, we propose and realize two complementary protocols to probe these degeneracies based on, respectively, symmetry-resolved entanglement entropies and measurement-based computational algorithms. The two protocols link quantum information processing to the classification of SPT phases of matter. They invoke the creation of a cluster state and are implemented on an IBM quantum computer. The experimental findings are compared to noisy simulations, allowing us to study the stability of topological states to perturbations and noise.
\end{abstract}

DOI: 10.1103/PhysRevLett.125.120502

One of the most important achievements in modern physics is the discovery and classification of topological phases of matter. Topological states do not break any local symmetry and, hence, are robust against local perturbations. In the context of quantum computation, this protection can be used to perform quantum protocols that are robust to local noise sources. The downside of this protection is that local probes are insufficient to identify topological states. Hence, even if one is able to create a topological state, demonstrating its topological character can be very challenging.

In this Letter, we address this question for a specific class of topological states, known as symmetry-protected topological (SPT) states. SPT phases can be identified by inspecting their entanglement spectrum (ES), i.e., the set of eigenvalues of the reduced density matrix of a subsystem $\rho_{A}$. In particular, for ground states of one-dimensional (1D) SPT phases, the ES is formed by degenerate pairs (or multiplets), while in topologically trivial states there is no protected degeneracy [1-4]. A simple explanation for the existence of ES degeneracies is offered by the symmetryresolved structure of $\rho_{A}[5,6]$. Consider a SPT phase

Published by the American Physical Society under the terms of the Creative Commons Attribution 4.0 International license. Further distribution of this work must maintain attribution to the author(s) and the published article's title, journal citation, and DOI. protected by a unitary symmetry $G=G_{A} \times G_{B}$, where $G_{A}$ and $G_{B}$ act on subsystems $A$ and $B$, respectively. Because $G$ commutes with the Hamiltonian, the ground state of the SPT phase $\left|\psi_{\mathrm{gs}}\right\rangle$ is an eigenstate of the symmetry operator $G$. When performing a partial trace $\rho_{A}=\operatorname{Tr}_{B}\left[\left|\psi_{\mathrm{gs}}\right\rangle\left\langle\psi_{\mathrm{gs}}\right|\right]$, the conservation of $G$ guarantees that $\rho_{A}$ is block diagonal in $G_{A}$, see Fig. 1. One can then define symmetry-resolved reduced density matrices as $\tilde{\rho}_{A}=\Pi_{A} \rho_{A} \Pi_{A}$, where $\Pi_{A}$ projects a state on a specific symmetry sector. For simple SPT states, like the Haldane phase of integer spins or Kitaev chains, it was found $[7,8]$ that $\tilde{\rho}_{A}$ that belong to different sectors are identical, leading to a degenerate ES [9].

A related property of SPT phases is the possibility to use their ground states as resources for measurement-based quantum computation (MBQC), where the process of computation is driven by local measurements [11-13]. A paradigmatic example of MBQC is offered by the quantumwire protocol, for which the goal is to transfer quantum

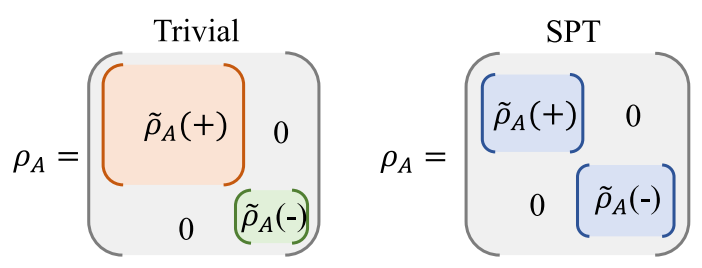

FIG. 1. Schematic distinction between trivial states and SPT ones. 
information between the two edges of a one-dimensional chain. In this protocol, the input state is implemented in the protected edge state of the SPT phase and measurements are used to progressively reduce the size of the chain and transfer the information to the opposite edge. Reference [13] established that the quantum-wire protocol is a uniform property of all ground states belonging to a given SPT phase of 1D spin chains. This result was subsequently extended to include measurement-based quantum gates in 1D SPT phases [14,15] and, finally, to universal MBQC in 2D SPT phases [16-19].

Here, we propose to use symmetry-resolved density matrices and MBQC protocols to identify the SPT properties of a quantum state. First, we develop and implement a new quantum protocol that accesses each symmetry sector individually. The equivalence of the different sectors helps us identify SPT states and distinguish them from trivial ones. Next, we extend the MBQC wire protocol [13] to include local perturbations. We demonstrate that the protocol can be disturbed only by perturbations that break the symmetry and make the state trivial, hence providing a complementary method to identify SPT states.

Cluster state.-Having in mind the physical realization of our algorithm using qubits, we focus on the 1D cluster Ising Hamiltonian

$$
H_{\text {cluster }}=-\sum_{i} h_{i}=-\sum_{i} Z_{i-1} X_{i} Z_{i+1}
$$

where $\{X, Y, Z\}$ are Pauli matrices and $h_{i}$ are referred to as stabilizers [12,20-31]. Its ground state, also known as the $1 \mathrm{D}$ cluster state $\left|\psi_{\text {cluster }}\right\rangle$, is a topological state protected by the $\mathbb{Z}_{2} \times \mathbb{Z}_{2}$ symmetry associated with the conservation of $P_{\text {odd }}=\prod_{i} h_{2 i+1}=\prod_{i} X_{2 i+1}$ and $P_{\text {even }}=\prod_{i} h_{2 i}=\prod_{i} X_{2 i}$. These operators correspond to parities on the sublattices of odd and even sites, respectively. For periodic boundary conditions, the reduced density matrix $\rho_{A}$ of the cluster state has four identical eigenvalues $\lambda=1 / 4$, one for each sector of the $\mathbb{Z}_{2} \times \mathbb{Z}_{2}$ symmetry (see Sec. D of the Supplemental Material [32]).

The Hamiltonian $H_{\text {cluster }}$ can be obtained from a trivial Hamiltonian $H_{\text {trivial }}=-\sum_{i} X_{i}$ by the transformation $X_{i} \rightarrow$ $Z_{i-1} X_{i} Z_{i+1}$ and $Z_{i} \rightarrow Z_{i}$ [33]. Similarly, $\left|\psi_{\text {cluster }}\right\rangle$ can be prepared in two steps [34,35]: (i) Hadamard gates that bring the system to the ground state of $H_{\text {trivial }},\left|\psi_{\text {trivial }}\right\rangle=\mid+$ $++\cdots\rangle$ and (ii) controlled- $Z$ gates that realize the transformation [see Fig. 2(a)] [36-40]. If the first and last qubits are not linked [see Fig. 2(a)], one obtains a system with open boundary conditions. In this case, the first and last terms of the corresponding Hamiltonian [see Eq. (1)] become $h_{1}=X_{1} Z_{2}$ and $h_{L}=Z_{L-1} X_{L}$ and the state conserves the total parity $P=(-1)^{L} \prod_{i=1}^{L} h_{i}=Y_{1} X_{2} X_{3}, \ldots$, $X_{L-1} Y_{L}$.

Symmetry-resolved entropies.-As mentioned in the Introduction, we use symmetry-resolved reduced density (a) $\left|\psi_{\text {cluster }}\right\rangle$ (b) Basic SWAP

(d) Quantum wire
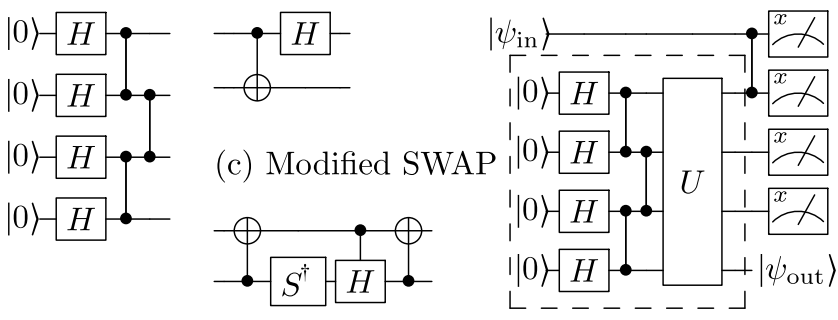

FIG. 2. Building blocks of the quantum circuits used in this Letter: (a) Preparation of the cluster state $\left|\psi_{\text {cluster }}\right\rangle$. (b) Basic SWAP test, which takes the singlet to $|11\rangle$ and the triplets to a mixture of $|00\rangle,|01\rangle,|10\rangle$, reproduced from Refs. [41,42]. (c) Modified SWAP test, which identifies all four eigenvectors of $\left(Z_{i} \otimes I\right)$ SWAP. This gate is used to compute symmetryresolved purities. (d) MBQC teleportation algorithm, using the state $U\left|\psi_{\text {cluster }}\right\rangle$ as a resource. See also Sec. F of the Supplemental Material [32] for the full quantum circuits.

matrices $\tilde{\rho}_{A}$ to identify the SPT nature of the cluster state. A direct measure of these matrices (state tomography) requires an exponentially large number of measurements. We overcome this difficulty by addressing the moments of these matrices, $\tilde{S}_{n}=\operatorname{Tr}\left[\tilde{\rho}_{A}^{n}\right]$, which can be measured by realizing $n$ copies of the state [43-52]. Specifically, for $n=2$, this approach is based on the identity

$$
\operatorname{Tr}\left[\rho^{2}\right]=\operatorname{Tr}[\rho \otimes \rho \mathrm{SWAP}] .
$$

Here, $\rho \otimes \rho$ is the combined state of two independently prepared copies of a state, and the operator SWAP swaps the states of the two copies. By applying the SWAP operator only to the subsystem $A$, one can compute the purity of $A, \operatorname{Tr}\left[\rho_{A}^{2}\right]$. Finally, if the SWAP operator is measured along with the projector to the conserved sectors, one can directly obtain the symmetry-resolved entropy $\tilde{S}_{n}$ [47,53-55].

To implement these ideas on a quantum computer, we create two copies of the cluster state with $L=4$ qubits, using twice the circuit of Fig. 2(a). Next, we measure the SWAP operator on each pair of qubits of the two copies, using the quantum circuit introduced by Refs. [41,42], see Fig. 2(b) (see also Supplemental Material [32]). By repeatedly measuring the output of the circuit, we infer the expectation values of the products of the SWAP operators of each site of a subsystem $A$, which correspond to $S_{2}=\operatorname{Tr}\left[\rho_{A}^{2}\right]$. In Fig. 3, we plot $-\ln S_{2}$, also known as the "second Rényi entropy", as a function of the subsystem size $L_{A}$. The result of this calculation matches the known properties of the cluster state with open boundary conditions: For any $0<L_{A}<L, \rho_{A}$ has two identical eigenvalues $\lambda=1 / 2$, one for each sector of the symmetry $P$, and one has $S_{2}=1 / 2$. Importantly, for $L_{A}=L$, one has $S_{2}=\operatorname{Tr}\left[\rho^{2}\right]=1$, indicating that the system is pure. 


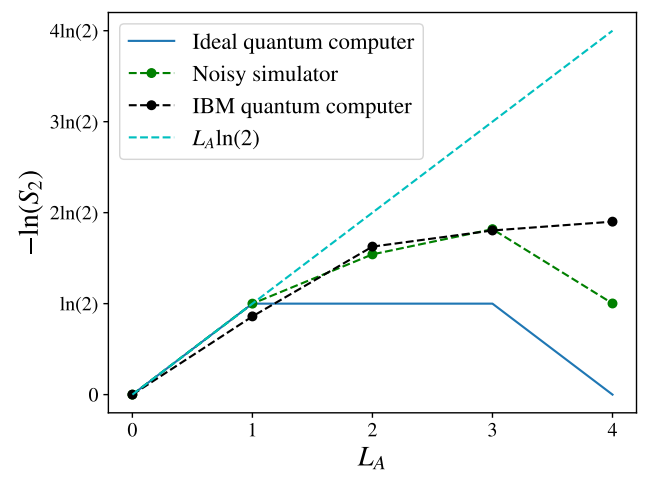

FIG. 3. Realization and characterization of a cluster state $\left|\psi_{\text {cluster }}\right\rangle$ with $L=4$ qubits. Second Rényi entropy as a function of the subsystem size $L_{A}$.

We now turn to symmetry-resolved measurements, which unveil the SPT nature of a state. The first moment, $\tilde{S}_{1}=\operatorname{Tr}\left[\tilde{\rho}_{A}\right]$, is simply the probability to find a subsystem in a specific sector of the symmetry. To compute the second moment, we develop a method to measure the product of the SWAP and $P$ operators, where $P$ acts on one copy only, see Fig. 2(c). This method can be generalized to richer symmetries with a larger number of topological phases; see Sec. A of the Supplemental Material [32] for details. The results of these calculations are shown in the upper row of Fig. 4: For the trivial state, the entire weight lies in the even parity sector, $P=+1$. For the cluster state, the full system $\left(L_{A}=L\right)$ is still an eigenvector of $P$ with $P=+1$. In contrast, smaller subsystems $\left(L_{A}<L\right)$ occupy with equal probabilities the sectors $P=+1$ and $P=-1$, in agreement with the topologically protected degeneracy of the symmetry-resolved reduced density matrices.

Noisy SPT states.-To understand actual experiments, it is necessary to study the effect of noise on topological states. Several earlier works addressed this question by extending the topological classification of pure states to density matrices [56-64]. Here, we focus on the effect of noise on the degeneracies of the ES, as probed by symmetry-resolved reduced density matrices. We define a noise source to be symmetry preserving if it preserves this degeneracy (and vice versa); see Refs. [65-67] and Sec. B of the Supplemental Material [32] for a formal definition.

Let us consider the results of a noisy simulation, obtained using QISKIT Aer [68]. The simulator computes the evolution of the density matrix by taking into account realistic noise sources in terms of Kraus operators. The parameters used in the simulation are determined by direct measurements of the success probability of the gates in the physical system [69]. Interestingly, all noise sources present in this simulation are symmetry preserving [70], with the exception of a measurement bias that leads to a systematic error toward zero outcomes. To study the effects of symmetry-preserving noise sources, we manually eliminate this bias from the simulations. In this case, if the system is prepared in a SPT state belonging to the same universality class as the cluster state, the noise does not lift the ES degeneracies.

We first consider the effects of noise on $S_{2}=\operatorname{Tr}\left[\rho^{2}\right]$, see Fig. 3. In the presence of noise, the state is not pure and the second Rényi entropy of the full system is $\approx \ln (2)$. This value is significantly smaller than the maximally allowed value of $4 \ln (2)$, indicating that the output of the simulation is not trivial. The slope of the entropy changes in the second half of the chain, as in the ideal quantum computer. To study the SPT properties of this noisy state, we compute symmetry-resolved quantities, see Fig. 4. For the trivial state, we find that both the probability and the symmetryresolved purity are larger for $P=+1$ than for $P=-1$. In contrast, in the cluster state, the probabilities and purities are identical for the two sectors for all $L_{A}<L$. (a) Probability $\tilde{S}_{1}=\operatorname{Tr}\left[\tilde{\rho}_{A}\right]$

Trivial

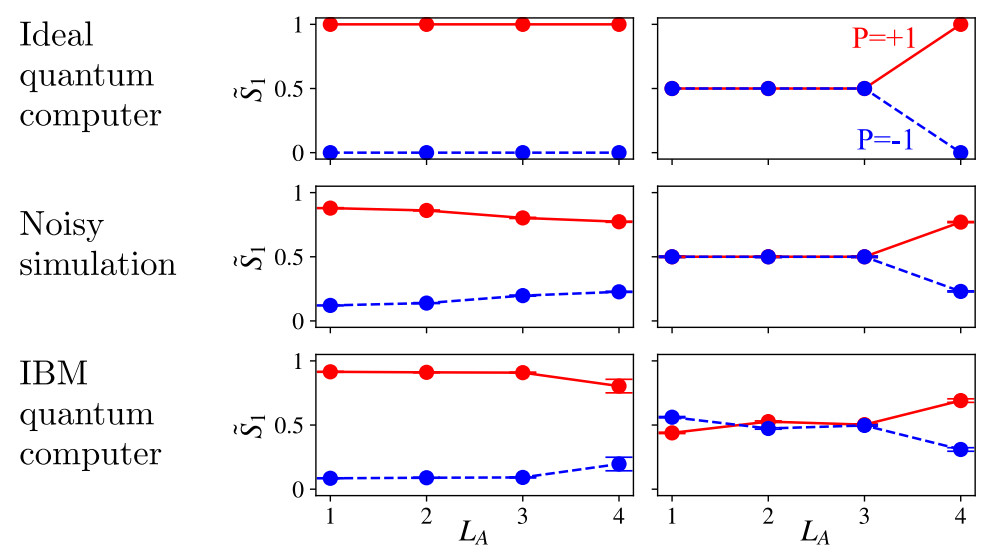

(b) Purity $\tilde{S}_{2}=\operatorname{Tr}\left[\tilde{\rho}_{A}^{2}\right]$

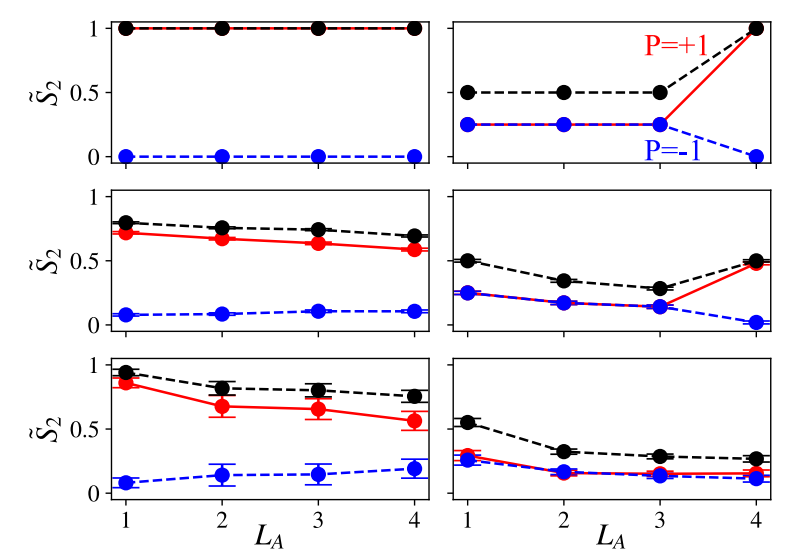

FIG. 4. Symmetry-resolved entanglement measures: (a) $\tilde{S}_{1}$ and (b) $\tilde{S}_{2}$, for the trivial state $\left|\psi_{\text {trivial }}\right\rangle$ and for the topological state $\left|\psi_{\text {cluster }}\right\rangle$. The $P=+1, P=-1$, and total contributions are shown in red, blue, and black, respectively. 
Remarkably, the total system $\left(L_{A}=L\right)$ is mostly in the $P=+1$ state, confirming that the system is targeting the correct pure state.

Using the same QISKIT package [68], we performed the same calculations on the 15-qubit Melbourne IBM quantum computer, using 150 runs with 8192 measurements each [71]. This computer has 15 qubits organized in a ladder structure, with physical 2-qubit gates between nearest neighbors only. This structure is ideal for the circuit under the present consideration: we realize the two copies of the cluster states on the two parallel chains that form the ladder and use the rungs to realize the SWAP operators. The results obtained in the actual computer are similar to those observed in the simulator: although the purity of the cluster state is not ideal, our symmetry-resolved probes still correctly identify its SPT nature. One interesting difference between the quantum computer and the noisy simulator can be observed in the symmetry-resolved probes of small subsystems, $L_{A}=1,2$. In the actual computer, the two sectors show small, but statistically significant, differences. We identify these errors as due to symmetry-breaking noise sources, such as the aforementioned measurement bias, which were absent in the simulation but present in the physical system. This bias also explains why the Rényi entropy of the $L_{A}=1$ subsystem [Fig. 3] is smaller than $1 / 2$ (see Sec. C of the Supplemental Material [32]). Our results demonstrate that topological arguments can be used to characterize the main sources of errors and classify them according to their symmetry.

Measurement-based wire protocol.-We now turn to the experimental realization of the symmetry-protected wire protocol [13]. In this protocol, a general quantum state is encoded in one boundary of the spin chain. The state is then shuttled to the other boundary in a teleportationlike fashion, by local measurements of the spins along the chain. We apply this protocol to a family of SPT states with $\mathbb{Z}_{2} \times \mathbb{Z}_{2}$ symmetry, which contains the $1 \mathrm{D}$ cluster state as a special case. All states in the family possess the same SPT order and, hence, have the same capacity to transmit 1-qubit worth of quantum information. Our goal is to verify the robustness of the protocol against variation within the phase.

For our implementation on an IBM quantum computer we use the $L=4$ cluster state $\left|\psi_{\text {cluster }}\right\rangle$ described above. The corresponding $\mathbb{Z}_{2} \times \mathbb{Z}_{2}$ symmetry is generated by $P_{\text {odd }}=\prod_{i=1,3} h_{i}=X_{1} X_{3} Z_{4}$ and $P_{\text {even }}=\prod_{i=2,4} h_{i}=$ $Z_{1} X_{2} X_{4}$, where $h_{i}$ are defined in Eq. (1). The family of SPT states is created applying either symmetry-preserving unitaries $U_{S}(\alpha, \beta)=e^{i \beta Z_{1} X_{2} Z_{3}} e^{i \alpha X_{3}}$ or symmetry-breaking unitaries $U_{\mathrm{SB}}(\alpha, \beta)=e^{i \beta Z_{1} X_{2} Z_{3}} e^{i \alpha Y_{3}}$ to $\left|\psi_{\text {cluster }}\right\rangle$. In the former case, all resource states respect the $\mathbb{Z}_{2} \times \mathbb{Z}_{2}$ symmetry and can be continuously connected in a symmetry-respecting fashion to the cluster state. In the latter case, the symmetry is broken and computational uniformity is not guaranteed.

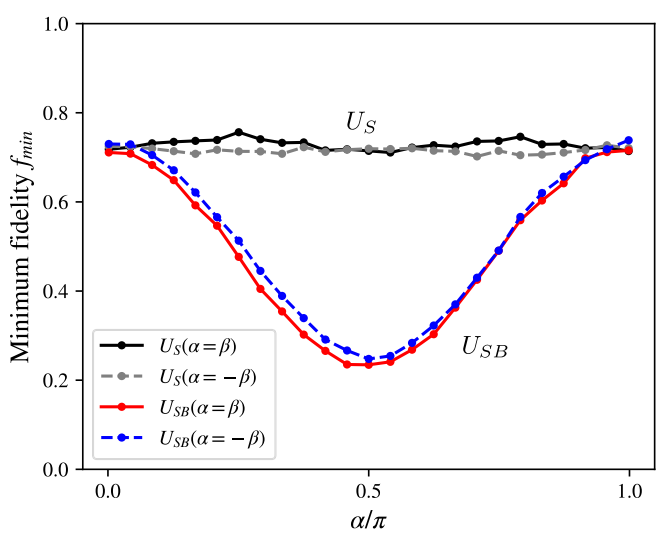

FIG. 5. Fidelity of the MBQC teleportation algorithm under the influence of symmetry-(non)preserving perturbations. Each data point represents the minimal fidelity with respect to six initial states (see Sec. E of the Supplemental Material [32] for the raw data).

Next, we introduce another qubit realizing the input state $\left|\psi_{\text {in }}\right\rangle$ and teleport it into the wire by performing a measurement in the 2-qubit cluster basis (a locally rotated Bell basis, $\{|+0\rangle \pm|-1\rangle\})$ on $\left|\psi_{\text {in }}\right\rangle$ and the first qubit of the spin chain, see Fig. 2(d). This particular measurement is chosen to be compatible with the MBQC wire protocol, consisting of local measurements in the $X$ basis of the remaining qubits and classically controlled Pauli correction depending on the measurement outcomes. Figure 5 shows the experimentally measured minimum fidelity $f_{\min }=$ $\min _{i}\left\langle\psi_{\text {in }}^{i}\left|\rho_{\text {out }}^{\exp }\right| \psi_{\text {in }}^{i}\right\rangle$ for six different input states $\left|\psi_{\text {in }}^{i}\right\rangle$ and the Pauli-corrected output state $\rho_{\text {out }}^{\exp }$ resulting from the wire protocol, for the choices $\beta= \pm \alpha$ in both the symmetric and the symmetry-breaking case; see also Sec. E of the Supplemental Material [32]. We find that the transmission fidelity is constant as a function of $\alpha$ in the symmetryrespecting case. In the symmetry-breaking case, the transmission fidelity is nonconstant as the resource state is varied.

Conclusion.-In this Letter, we devised and implemented experimentally two methods to identify the SPT nature of the cluster state on a quantum computer. The first algorithm stems from the observation that in SPT states, the reduced density matrix $\rho_{A}$ is formed by identical blocks that correspond to different sectors of the underlying symmetry. The flexibility of the quantum computer makes it possible to directly probe the moments of density matrices by projecting the quantum state into the different symmetry sectors. The realization of this algorithm on both a quantum simulator and on an IBM quantum computer allowed us to study the impact of time-dependent noise on the SPT order of the state. In particular, we found that, while most of realistic noise sources are symmetry preserving, the systematic measurement bias of the physical machine breaks this symmetry. Its effects are, however, small enough to enable us to identify the SPT nature of the cluster state. 
The second way to characterize the SPT order of the cluster states consists of using them as a buffer for MBQC teleportation. The fidelity of this protocol is unaffected by symmetry-preserving terms, and vice versa for symmetrybreaking terms, allowing us to identify the SPT order of the cluster states. The equivalence between these two methods demonstrates a deep relation between two separate fields of science - condensed matter physics and quantum information theory.

Our work has important implications for the modeling of noisy intermediate-scale quantum computers. We demonstrated that topological arguments are an efficient tool to identify and classify noise sources in quantum computers. This information can be used to improve the performance of quantum computers, for example, by gauging the measurement apparatus to take into account systematic errors. From a fundamental perspective, we identified sufficient conditions under which a noisy quantum state can retain its SPT properties. This aspect may have implications for quantum computations: for pure states, it was shown that the classification of SPT phases is in one-to-one correspondence with the possibility to use it as a resource for one-way quantum computers. Although this question deserves further investigation, we conjecture that this link extends to noisy systems as well.

We acknowledge useful discussions with Yael BenHaim, Moshe Goldstein, Joe Jackson, Yuval Tamir, Ari Turner, Hannes Pichler, and Frank Pollmann. We acknowledge support from ARO (W911NF-20-1-0013) (R. R. and E. S.). This work is supported by the Israel Science Foundation, Grants No. 151/19 (D. A. and E. G. D. T.) and No. 154/19 (D. A., Y. N., E. S., and E. G. D. T.). We acknowledge the use of IBM Q Experience for this work. The views expressed are those of the authors and do not reflect the official policy or position of IBM or the IBM Quantum team.

*Present address: Classiq Technologies.

[1] F. Pollmann, A. M. Turner, E. Berg, and M. Oshikawa, Phys. Rev. B 81, 064439 (2010).

[2] L. Fidkowski, Phys. Rev. Lett. 104, 130502 (2010).

[3] X. Chen, Z.-C. Gu, Z.-X. Liu, and X.-G. Wen, Phys. Rev. B 87, 155114 (2013).

[4] The degeneracy of the ES of a SPT state is exact only in the thermodynamic limit where both the system size and the size of the subsystem tend to infinity. For finite size systems, the degeneracy is exponentially suppressed as the ratio between the (sub)system size and the correlation length of the state.

[5] N. Laflorencie and S. Rachel, J. Stat. Mech. (2014) P11013.

[6] M. Goldstein and E. Sela, Phys. Rev. Lett. 120, 200602 (2018).

[7] E. Cornfeld, L. A. Landau, K. Shtengel, and E. Sela, Phys. Rev. B 99, 115429 (2019).
[8] S. Fraenkel and M. Goldstein, J. Stat. Mech. (2020) 033106.

[9] A general relationship between symmetry sectors $\tilde{\rho}_{A}$ in arbitrary SPT states can be derived using cohomology theory [10].

[10] D. Azses and E. Sela, arXiv:2008.09332.

[11] A. Miyake, Phys. Rev. Lett. 105, 040501 (2010).

[12] A. C. Doherty and S. D. Bartlett, Phys. Rev. Lett. 103, 020506 (2009).

[13] D. V. Else, I. Schwarz, S. D. Bartlett, and A. C. Doherty, Phys. Rev. Lett. 108, 240505 (2012).

[14] J. Miller and A. Miyake, Phys. Rev. Lett. 114, 120506 (2015).

[15] R. Raussendorf, D.-S. Wang, A. Prakash, T.-C. Wei, and D. T. Stephen, Phys. Rev. A 96, 012302 (2017).

[16] R. Raussendorf, C. Okay, D.-S. Wang, D. T. Stephen, and H. P. Nautrup, Phys. Rev. Lett. 122, 090501 (2019).

[17] T. Devakul and D. J. Williamson, Phys. Rev. A 98, 022332 (2018).

[18] D. T. Stephen, H. P. Nautrup, J. Bermejo-Vega, J. Eisert, and R. Raussendorf, Quantum 3, 142 (2019).

[19] A. K. Daniel, R. N. Alexander, and A. Miyake, Quantum 4, 228 (2020).

[20] D. Gottesman, arXiv:quant-ph/9705052.

[21] H. J. Briegel and R. Raussendorf, Phys. Rev. Lett. 86, 910 (2001).

[22] J. Keating and F. Mezzadri, Commun. Math. Phys. 252, 543 (2004).

[23] A. Kopp and S. Chakravarty, Nat. Phys. 1, 53 (2005).

[24] W. Son, L. Amico, R. Fazio, A. Hamma, S. Pascazio, and V. Vedral, Europhys. Lett. 95, 50001 (2011).

[25] P. Smacchia, L. Amico, P. Facchi, R. Fazio, G. Florio, S. Pascazio, and V. Vedral, Phys. Rev. A 84, 022304 (2011).

[26] Y. Niu, S. B. Chung, C.-H. Hsu, I. Mandal, S. Raghu, and S. Chakravarty, Phys. Rev. B 85, 035110 (2012).

[27] W. DeGottardi, M. Thakurathi, S. Vishveshwara, and D. Sen, Phys. Rev. B 88, 165111 (2013).

[28] V. Lahtinen and E. Ardonne, Phys. Rev. Lett. 115, 237203 (2015).

[29] T. Ohta, S. Tanaka, I. Danshita, and K. Totsuka, Phys. Rev. B 93, 165423 (2016).

[30] T. E. Lee, Y. N. Joglekar, and P. Richerme, Phys. Rev. A 94, 023610 (2016).

[31] B.-E. Friedman, A. Rajak, and E. G. Dalla Torre, Europhys. Lett. 125, 10008 (2019).

[32] See Supplemental Material at http://link.aps.org/ supplemental/10.1103/PhysRevLett.125.120502 for all circuits used in this Letter.

[33] Incidentally, we note that by applying a similar transformation $X_{i} \rightarrow Z_{i-1} X_{i} Z_{i+1}$ and $Y_{i} \rightarrow Z_{i}$ (with additional phase factors) multiple times, one obtains a series of topological states of increasing complexity [10,31].

[34] K. Choo, C. W. von Keyserlingk, N. Regnault, and T. Neupert, Phys. Rev. Lett. 121, 086808 (2018).

[35] A. Smith, B. Jobst, A. G. Green, and F. Pollmann, arXiv: 1910.05351.

[36] N. H. Lindner and T. Rudolph, Phys. Rev. Lett. 103, 113602 (2009).

[37] S. E. Economou, N. Lindner, and T. Rudolph, Phys. Rev. Lett. 105, 093601 (2010). 
[38] I. Schwartz, D. Cogan, E. R. Schmidgall, Y. Don, L. Gantz, O. Kenneth, N. H. Lindner, and D. Gershoni, Science 354, 434 (2016).

[39] D. Buterakos, E. Barnes, and S. E. Economou, Phys. Rev. X 7, 041023 (2017).

[40] H. Pichler, S. Choi, P. Zoller, and M. D. Lukin, Proc. Natl. Acad. Sci. U.S.A. 114, 11362 (2017).

[41] J. C. Garcia-Escartin and P. Chamorro-Posada, Phys. Rev. A 87, 052330 (2013).

[42] L. Cincio, Y. Subaşı, A. T. Sornborger, and P. J. Coles, New J. Phys. 20, 113022 (2018).

[43] A. J. Daley, H. Pichler, J. Schachenmayer, and P. Zoller, Phys. Rev. Lett. 109, 020505 (2012).

[44] H. Pichler, L. Bonnes, A. J. Daley, A. M. Läuchli, and P. Zoller, New J. Phys. 15, 063003 (2013).

[45] R. Islam, R. Ma, P. M. Preiss, M. E. Tai, A. Lukin, M. Rispoli, and M. Greiner, Nature (London) 528, 77 (2015).

[46] S. Johri, D. S. Steiger, and M. Troyer, Phys. Rev. B 96, 195136 (2017).

[47] E. Cornfeld, M. Goldstein, and E. Sela, Phys. Rev. A 98, 032302 (2018).

[48] E. Cornfeld, E. Sela, and M. Goldstein, Phys. Rev. A 99, 062309 (2019).

[49] R. Bonsignori, P. Ruggiero, and P. Calabrese, J. Phys. A 52, 475302 (2019).

[50] N. Feldman and M. Goldstein, Phys. Rev. B 100, 235146 (2019).

[51] S. Murciano, G. Di Giulio, and P. Calabrese, SciPost Phys. 8, 046 (2020).

[52] M. T. Tan and S. Ryu, Phys. Rev. B 101, 235169 (2020).

[53] J. C. Xavier, F. C. Alcaraz, and G. Sierra, Phys. Rev. B 98, 041106(R) (2018).

[54] H. Barghathi, E. Casiano-Diaz, and A. Del Maestro, Phys. Rev. A 100, 022324 (2019).

[55] Mathematically, a proof of the equivalence between the symmetry sectors requires one to access the full ES, demanding a number of measurements that grows with the size of the Hilbert space. However, in practice, it is often sufficient to check that the symmetry exists in the first few moments to demonstrate the SPT nature of the state.

[56] S. Diehl, E. Rico, M. A. Baranov, and P. Zoller, Nat. Phys. 7, 971 (2011).

[57] O. Viyuela, A. Rivas, and M. A. Martin-Delgado, Phys. Rev. Lett. 112, 130401 (2014).

[58] E. P. L. van Nieuwenburg and S. D. Huber, Phys. Rev. B 90, 075141 (2014).

[59] O. Andersson, I. Bengtsson, M. Ericsson, and E. Sjöqvist, Phil. Trans. R. Soc. A 374, 20150231 (2016).

[60] D. Linzner, L. Wawer, F. Grusdt, and M. Fleischhauer, Phys. Rev. B 94, 201105(R) (2016).

[61] Z. Gong, Y. Ashida, K. Kawabata, K. Takasan, S. Higashikawa, and M. Ueda, Phys. Rev. X 8, 031079 (2018).

[62] C.-E. Bardyn, L. Wawer, A. Altland, M. Fleischhauer, and S. Diehl, Phys. Rev. X 8, 011035 (2018).

[63] M. Goldstein, SciPost Phys. 7, 067 (2019).

[64] M. Asorey, P. Facchi, and G. Marmo, Open Syst. Inf. Dyn. 26, 1950012 (2019).

[65] B. Buča and T. Prosen, New J. Phys. 14, 073007 (2012).

[66] B. Baumgartner and H. Narnhofer, J. Phys. A 41, 395303 (2008).

[67] V. V. Albert and L. Jiang, Phys. Rev. A 89, 022118 (2014).

[68] H. Abraham et al., QISKIT: An open-source framework for quantum computing, 2019, https://zenodo.org/record/ 2562111.

[69] We used the noise model that matches the experiments' date.

[70] qiskit/providers/aer/noise/errors/standard_errors.py, https:// github.com/Qiskit/qiskit-aer/blob/d8d77270c745e4c31129ce7f816a93e1efc2e743/qiskit/providers/aer/noise/errors/ standard\_errors.py, accessed Nov. 21, 2019.

[71] The experiments were performed on Dec. 26-28, 2019. 